\title{
EDIFICATE
}

I Congreso de Escuelas de Edificación y Arquitectura Técnica de España

València, 4 y 5 de noviembre de 2021

Escuela Técnica Superior de Ingeniería de Edificación

Universitat Politècnica de València

Doi: https://doi.org/10.4995/EDIFICATE2021.2021.13550

\section{La ingeniería de edificación. Una apuesta de futuro Building engineering. Looking forward}

\author{
Juan M. Santiago Zaragoza ${ }^{a}$, Fabián García Carrillob y Joaquín Passolas Colmeneroc, \\ Emilio Gómez Cobos ${ }^{d}$ \\ apresidente CODATIE, Escuela Técnica Superior de Ingeniería de Edificación, Universidad de \\ Granada, santi@ugr.es, bFabián García Carrillo, Coodinador del Grado, Escuela Técnica Superior de \\ Ingeniería de Edificación, Universidad de Granada, fabian@ugr.es y ' Coordinador del Libro Blanco de \\ Ingeniería de Edificación, Escuela Técnica Superior de Ingeniería de Edficación, Universidad de \\ Granada, passolas@ugr.es. ${ }^{d}$ Escuela Técnica Superior de Ingeniería de Edficación, Universidad de \\ Granada, emiliog@ugr.es
}

\begin{abstract}
Building Engineering White Paper, was a turning point on the transformation of the study plan linked to Arquitecto Técnico profession that, in Spain is a regulated one. A simple search in the European catalog of University Titles that can be extended to the whole World, give a zero result about that professional name. So, analyzing that catalog, or this Title is included within Surveyors or Project Managers that means studies of Vocational Training, or is included within Building Engineers as a University Degree. The latter means an automatic recognition in most of the World countries that lead to the chosen designation of Building Engineering for the Study Plan.
\end{abstract}

It is well known the surprising Supreme Court Sentence (SCS) of 2010 that canceled this designation in some universities. That meant a helplessness situation for universities self-government established in the Spanish Constitution (art. 27).

Nevertheless under various names, that include Building Engineering, the different Study Plans (without doubt of the Engineering Field) are in force. The access to Postgraduates Studies with a wide offer of Masters in different universities (with no additional courses) and Doctorate Programs, was a milestone in the History of that Title that lead to a fundamental transformation of our Faculties.

In the field of Knowledge, the "Glass Ceiling", that required to study different careers to achieve a better academic and professional category, was broken. 
Many agreements with foreign universities and double Grade titles, mainly with European Universities certify our international recognition, most of the occasions easier than in our country universities.

Coming back to the designation of Building Engineering is looking forward to the future.

Keywords: white paper, building engineering, sustainable construction

\section{Resumen}

El Libro Blanco de Ingeniería de Edificación, supuso un antes y un después en la transformación de los estudios vinculados con la profesión, de actividad regulada en España, de Arquitecto Técnico. Una búsqueda simple, en el catálogo de titulaciones universitarias europeas y mundiales, arroja un resultado nulo, sobre la base de la denominación de este título español. Analizado dicho catálogo, o se incluye dicha titulación en el entorno de la agrimensura y la gestión de proyectos, lo que supondría una clara contracción del ámbito y nivel de formación, de su actividad real, y llevaría a la consiguiente degradación entre la Formación Profesional, en los Ciclos Superiores. O se incluye en el ámbito de la Ingeniería de Edificación, lo que supone un encaje real de la formación y la profesión desarrollada, y un reconocimiento automático en el resto del mundo. De ahí que en su momento, al albur de la construcción del EEES, desde la Universidad se optara por la denominación más cierta y reconocible de Ingeniería de Edificación.

De todos es conocida la sorprendentes y contradictorias STS de 2010, por las que, solo en determinadas universidades, se anulaba la denominación del título de Grado en Ingeniería de Edificación, no solo aumentando la presunta "confusión" que, se suponía resolver, generando incertidumbre para los antiguos y nuevos titulados europeos, y además, dejando a la Universidad española en situación de indefensión en lo que a su autonomía universitaria se refiere (art. 27 C.E.).

No obstante, esta es y será una profesión imprescindible, y hoy, con diversas denominaciones, que incluyen la de Ingeniería de Edificación, los planes de estudio del Libro Blanco de 2007, siguen vigentes y, sin lugar a dudas, formando a universitarios en el ámbito de la ingeniería, esto es, en la máxima capacitación para la resolución de problemas en el sector de la Edificación. El paso a la formación de posgrado, con numerosos másteres de especialización a los que se puede acceder (ya sin complementos de formación) y doctorados propios, suponen un reconocimiento de ello, un hito en la historia de la titulación renovada y una transformación radical de las Escuelas. 
En el ámbito del conocimiento, no hay límite que se circunscriba al de un ejercicio parcial y localmente regulado. Se rompió el famoso "techo de cristal" que obligaba a grandes esfuerzos personales para acceder a la formación en otras titulaciones, si se quería escalar en la categoría académica $y$ profesional.

Todo ello ha permitido alcanzar numerosos convenios con universidades extranjeras y acuerdos de doble titulación, sobre todo con universidades europeas, que valoran y acreditan ese reconocimiento y encaje internacional, tanto de la formación que ofrecen nuestras Escuelas, como de la profesión que ejercen nuestros titulados. Reconocimiento académico que, paradójicamente, en muchas ocasiones, es más fácil y directo que en nuestras propias y miopes universidades.

La vuelta a la denominación de la Ingeniería de Edificación es un reto de futuro por el que no se puede dejar de apostar.

Palabras clave: Libro Blanco. Ingeniería de Edificación. Construcción Sostenible.

\section{Introducción}

\subsection{EI Proceso de Bolonia. La nueva realidad del EEES}

En la década de los 80 del siglo XX se inició una reforma a nivel europeo que pretendía:

- Fomentar la competitividad internacional y

- Promover el empleo de los estudiantes europeos.

En 1988 se firmó la Carta Magna de las Universidades Europeas, suscrita por los rectores que se reunieron en Bolonia con motivo del 900 aniversario de su creación.

En Junio de 1999, once años después, se produce la Declaración de Bolonia, declaración conjunta de los Ministros Europeos de Educación reunidos en la citada capital italiana. Esta declaración tuvo su antecedente en la Declaración de la Sorbona, de mayo de 1998 que fue una declaración conjunta, en este caso para iniciar el proceso para la armonización del diseño del Sistema de Educación Superior Europeo (a cargo de los ministros de Francia, Alemania, Italia y Reino Unido).

El Proceso de Bolonia pretendió dar mayor coherencia a los sistemas de educación superior en Europa.

En una Europa unida en lo económico, la movilidad laboral se consideró como elemento imprescindible para conseguir la integración de los ciudadanos en cualquier país independientemente del país de procedencia. Para ello el Espacio Europeo de Educación Superior facilitó la movilidad de estudiantes y personal, haciendo que la educación superior 
fuera más inclusiva y accesible y, simultáneamente, con el objetivo de lograr que la educación superior en Europa fuera más atractiva y competitiva a escala mundial.

Como parte del Espacio Europeo de Educación Superior (EEES), todos los países participantes acordaron:

- Introducir un sistema de educación superior de tres ciclos, consistente en estudios de grado, máster y doctorado

- Garantizar el reconocimiento mutuo de las cualificaciones y los períodos de aprendizaje en el extranjero completados en otras universidades.

- Aplicar un sistema de garantía de la calidad, a fin de reforzar la calidad y pertinencia del aprendizaje y la enseñanza.

Ello dio lugar a un proceso de transformación de los modelos universitarios de los distintos países para adaptarse al EEES.

La adaptación al EEES generó un cambio de óptica que afectó no sólo el procedimiento de diseño de los nuevos planes y a la organización de los contenidos, sino que fue mucho más allá, e implicó cambios radicales en las metodologías de enseñanza y evaluación que se habían desarrollado hasta ese momento en el marco de la universidad española (Pallisera Díaz et al. 2010).

\subsection{El cambio en el sistema universitario español. Las nuevas titulaciones.}

Con objeto de proceder al cambio necesario de los distintos títulos del sistema universitario español, se produjo una convocatoria de la Agencia Nacional de Evaluación de la Calidad y Acreditación (ANECA) para la elaboración de los correspondientes Libros Blancos de los diferentes Títulos de Grado. El trabajo fue llevado a cabo por una red de universidades españolas, apoyadas por la ANECA, con objeto de realizar estudios y supuestos prácticos útiles en el diseño de las bases para un título de grado adaptado al EEES.

Por lo que a nuestra titulación respecta, la entonces Escuela Universitaria de Arquitectura Técnica, de la Universidad de Granada, con su director al frente, el profesor Joaquín Passolas Colmenero, coordinó el proyecto de Libro Blanco para el nuevo Título de Grado en Ingeniería de Edificación. En dicho proyecto participaron constituidas en Pleno, las 32 universidades que en aquel momento impartían la titulación de Arquitectura Técnica, y se invitó a un representante del Consejo General de la Arquitectura Técnica, el colectivo profesional.

Las 7 universidades coordinadoras-redactoras del Libro Blanco crearon cinco grupos de trabajo para dar contenido a los 14 puntos o secciones del proyecto:

1. Análisis de los estudios correspondientes o afines en Europa. Modelos y Centros contactados.

2. Los estudios en España. Planes de estudios vigentes. Estadísticas.

3. Los titulados y su inserción laboral. Perfiles profesionales.

4. Formación disciplinar y profesional. Competencias. 
5. El nuevo Título. Objetivos, estructura y créditos ECTS. Evaluación.

El proyecto así elaborado finalmente fue aprobado, por consenso general, en la última reunión del Plenario celebrada en la Escuela Universitaria de Arquitectura Técnica de la Universidad de Granada, el día 8 de junio de 2004.

A la estructura o bases académicas, se uniría el encaje administrativo. El 21 de diciembre de 2006, el Ministerio de Educación y Ciencia publicó un documento de trabajo de Propuesta de Directrices para la elaboración de títulos universitarios de grado y máster.

Finalmente, el Ministerio de Educación y Ciencia publicó el Real Decreto 1393/2007, de 29 de octubre, por el que se establece la ordenación de las enseñanzas universitarias oficiales. De acuerdo con dicha normativa las titulaciones en España se estructuraron de la siguiente forma (Pallisera Díaz et al 2010):

1. Los títulos de grado pasaron, con carácter general, a tener 240 créditos ECTS, con una duración de 4 años. Desaparecieron las licenciaturas y diplomaturas. En ese sentido la titulación de Arquitecto Técnico, de 3 cursos académicos más el Proyecto de Fin de Carrera, amplió su duración en un año suplementario.

2. Desapareció el catálogo de títulos, que era un listado limitado propuesto por los ministerios que tenían competencias universitarias, y se establecieron las competencias y contenidos de titulaciones con directrices europeas. Ello permitió a las universidades ejercer la autonomía en el sentido de definir nuevos títulos para dar respuesta a las nuevas necesidades sociales y del sistema productivo.

3. Otra de las características del nuevo modelo fue la formulación de nuevas titulaciones a partir de las competencias académicas, auténtica novedad en el sistema español, que tradicionalmente se basaba en las materias.

4. Por último se estableció un proceso riguroso de verificación y acreditación de los títulos universitarios, consistente en la aprobación inicial del título a través de la memoria de verificación, VERIFICA, y la posterior necesidad de renovación de la acreditación a los seis años de su aprobación, y renovaciones posteriores cada cuatro años

\section{Hacia una base común. La Ingeniería de Edificación en Europa}

Como se ha apuntado previamente, uno de los principales elementos estudiados por los grupos de trabajo del Libro Blanco de Ingeniería de Edificación, fue determinar la estructura de titulaciones y profesiones europeas relacionadas con la Arquitectura Técnica. Ante las preguntas, ¿Quién desempeña competencias análogas a nosotros en Europa?, ¿Cómo y dónde se forman?, y ¿Cómo se denominan dichas titulaciones y profesiones?, se acudió, actualizándolo a 2004, al anterior estudio "Las Profesiones del Sector de la Construcción en Europa”, elaborado, por el Centro de Documentación Josep Renart, del Colegio de Barcelona.

La respuesta, buscada en los principales países europeos, fue origen de los dos primeros capítulos del Libro Blanco: 1. Análisis de la situación de los estudios correspondientes o 
afines en Europa; y 2. Modelos de estudios europeos seleccionados y beneficios directos que aportará a los objetivos del Título la armonización que se propone.

En el primero de ellos se hizo un amplio recorrido por 15 países: Alemania, Austria, Bélgica, Dinamarca, Finlandia, Francia, Grecia, Irlanda, Italia, Luxemburgo, Noruega, Países Bajos, Portugal, Reino Unido y Suecia. De todos ellos se hizo un análisis de la formación académica y su denominación, el acceso al mundo profesional de los titulados, las funciones desempeñadas y la regulación legal del ejercicio profesional.

En el segundo capítulo, del análisis anterior, se identificaron algunas particularidades coincidentes, por sus tradiciones culturales, condiciones socio-económicas, peculiaridades geográficas, o simplemente criterios de homologación previamente adoptados, lo que permitió hacer una clasificación de índole territorial que llevaría a agruparlos, subdividiendo el espacio europeo estudiado (los países de Este aún no se habían incorporado) en cuatro áreas o zonas de influencia o de características comunes, que serían:

1. Zona escandinava, que agruparía a los países nórdicos, Dinamarca, Finlandia, Noruega y Suecia, de larga tradición compartida.

2. Zona anglosajona, que incluiría a los países que forman las Islas Británicas, Irlanda y Gran Bretaña, distinguiéndose entre Inglaterra, Gales y Escocia en el último caso.

3. Zona mediterránea, que además de España, incluiría a Portugal, Francia, Italia y Grecia. Aunque, desde un punto de vista educativo hay cierta divergencia.

4. Zona centroeuropea, que bajo la influencia de Alemania, incluiría también a Austria, Bélgica, Holanda y Luxemburgo.

Una primera conclusión fue comprobar que la formación y las funciones que desarrollaban los titulados europeos afines en el sector, en relación con lo que aquí tendría que ser una titulación homologable en el EEES, se enmarcaban en el campo común de actividad de la producción de la edificación, con bastante coincidencia entre ellos, por sectores culturales y áreas de influencia de los distintos países. $\mathrm{Y}$, asimismo, que las mayores correspondencias competenciales de nuestra formación se mantenían, y con un alto nivel de concurrencia, con determinadas titulaciones, ya asentadas en la mayoría de los países, en un ámbito de conocimiento y acción especifico, cual es la Ingeniería directamente vinculada con la construcción de edificios (Building Engineering, en su traducción común al inglés). Sólo en aspectos puntuales y en algunos países, se podría establecer una cierta relación con la Ingeniería de las Infraestructuras públicas y la Obra Civil, y menos aún con la Ingeniería Industrial. Con respecto al proceso de homologación se comprobó que los países de la zona mediterránea, aun con las mayores divergencias internas, serían los que deberían dar un mayor paso de acercamiento hacia los modelos ya implantados mayoritariamente en el resto de la Europa estudiada.

Finalmente, por lo que respecta a los centros de formación y a la denominación de los estudios afines, se pudo hacer el siguiente resumen de aquel estudio, del que surgió, mayoritariamente, la opción de "Ingeniería de Edificación" para equiparar a los nuevos 
estudios de grado españoles, y consecuentemente, identificar entre los ingenieros de edificación (building engineer), tanto a los titulados, como a los profesionales. A saber:

\subsection{Alemania}

Aquí, el título académico básico es de cuatro años, y se diferencia, claramente, según sea el tipo de establecimiento donde se cursan los estudios: bien sean las universidades científicas o Wissenschaftliche Hochschulen; bien sean las llamadas Fachhochschulen, término traducible, literalmente, por universidades de disciplinas, de orientación más profesionalizante. Si bien, el valor profesional de las titulaciones tiende a equipararse progresivamente, en base al ejercicio profesional desarrollado.

En Alemania se distinguen los siguientes títulos relacionados: Diplom-Ingenieur Architektur, o Ingeniero Diplomado en Arquitectura, procedente de una universidad científica, o bien Diplom-Ingenieur (FH) Fachrichtung Architektur, titulado en una Fachhochschule. Y más relacionados aún: Diplom-Ingenieur Fachrichtung Bauwesen, o Ingeniero Diplomado en Construcción, formado en una universidad científica, o bien Diplom-Ingenieur (FH) Fachrichtung o Ingeniero Graduado especializado, titulado en una Fachhochschule.

\subsection{Austria}

Los estudios universitarios relacionados con la edificación siguen la estela de Alemania, aunque su duración media suele ser algo superior y la formación es muy personalizada. Así, se ofertan titulaciones universitarias análogas, tanto en la distinción de centros de formación, como en la orientación profesional, distinguiéndose: Ingeniero Diplomado en Arquitectura por universidades técnicas, Diplom-Ingenieur Architektur (Technicche Hochschule); Y sobre todo: Ingeniero Diplomado de la Construcción por universidades técnicas, Diplom-Ingenieur Bauingenieurwesen (Technische Hochcchule). Existe una amplia oferta de especialidades, como: Ingeniería Constructiva - Konsfruktiver Ingenieurbau o Empresa Constructora y Economía de la Construcción - Baubetneb und Bauwirtschnft.

\subsection{Bélgica}

La Enseñanza Superior aquí tiene también dos orientaciones: la Enseñanza Superior universitaria, que incluye, en particular, los estudios de Ingeniería Civil ligados a la construcción; y la Enseñanza Superior no universitaria, que cubre otros títulos relacionados con dicho sector. Los estudios tienen una duración de 4 ó 5 años y se dividen en dos ciclos. El primero permite acceder al título de Candidato en la carrera considerada, pero solo el segundo da acceso a la profesión. Se pueden distinguir cuatro titulaciones de ciclo largo relacionadas: Arquitecto, Ingeniero Civil-Arquitecto, y, sobre todo, Ingeniero Civil de la Construcción, e Ingeniero Industrial de la Construcción.

\subsection{Dinamarca}

Las instituciones de Enseñanza Superior gozan de una gran autonomía para fijar los programas docentes, pero el ministerio competente establece las condiciones de acceso a 
los estudios y los requisitos para la obtención de los títulos. En la Enseñanza Superior universitaria se distinguen dos carreras relacionadas, de tipo largo, de 5 años o más: Arquitecto (que también puede ser diplomado de la Academia de Bellas Artes), e Ingeniero Civil. En la Enseñanza Superior de carácter no universitario, que se imparte en los llamados Hojere Loereanstalter, se icluyen otras carreras, de tipo intermedio 3 años y medio, entre las que se encuentra la que se puede traducir por Constructor o Arquitecto Constructor.

\subsection{Finlandia}

En Finlandia existen dos profesiones dedicadas a la construcción que se estudian en las universidades técnicas (Teknillinen Korkeakoulu), de una duración mínima de cuatro años y medio: Arquitecto - Aukkithefi, e Ingeniero Civil - Diploma Incinööri (Rakennustekniikka), título que se traduce literalmente por Ingeniero Diplomado (Técnica de la construcción). Existen otras dos profesiones, más próximas a la ejecución de las obras, que se forman en las Escuelas Politécnicas, asimismo son carreras de cuatro años de duración: Arquitecto Constructor - Rakennusarkkitehti, literalmente Arquitecto de la Construcción; e Ingeniero de la Construcción - Rakennusinsinööri. Aunque en Finlandia se emplea también la traducción inglesa Building Engineer, equiparando este título al nivel académico anglosajón Bachelor of Science.

\subsection{Francia}

En Francia la universidad ha mantenido, tradicionalmente, una fuerte independencia y una orientación esencialmente académica, no necesariamente vinculada a salidas profesionales concretas. Esto explica, en parte, el desarrollo, en paralelo a la enseñanza universitaria, de un complejo sistema de estudios y escuelas superiores especializadas, donde se forma a profesionales como ingeniero o arquitecto. Destacan las llamadas Grandes Ecoles (Escuelas Superiores de Ingeniería). En nuestro sector, aparte de otras titulaciones de menor duración y orientación profesionalizante, simplificadamente, se pueden distinguir dos carreras de tipo largo (5 años) relacionadas: Arquitecto, y sobre todo Ingeniero, donde existe la especialidad de Ingenieur de Travaux de la Construcción, orientado tanto a las obras públicas, como al sector de la construcción residencial.

\subsection{Grecia}

Se distingue entre la Enseñanza Superior que se imparte en universidades, institutos politécnicos y otras instituciones independientes, y la de carácter no universitario, que se imparte en los Institutos de Enseñanza Superior Técnica (TEI). En cuanto a los estudios superiores del sector de la edificación relacionados, existen dos carreras de tipo largo (5 años): Arquitecto, e Ingeniero con especialidades en Ingeniería Civil (Electricidad, Mecánica o Topografía). Asimismo existe una titulación traducible por Ingniero Técnico de 3,5 años.

\subsection{Irlanda}

Existe una gama relativamente amplia de formaciones especializadas relacionadas con el sector de la construcción. A nivel de graduados (Degree courses) se pueden destacar: 
Licenciado en Arquitectura - Bachelor of Architecture Degree, de cinco años reconocida profesionalmente en Irlanda y el Reino Unido; y Licenciado en Ingeniería Civil - Bachelor of Engineering (Civil), de cuatro años de duración.

En relación con la construcción, destacan las siguientes especializaciones: Ingeniería Estructural y Técnica Constructiva; Instalaciones y Control Ambiental en Edificios; Sistemas Informáticos y Gestión de la Construcción; y Diplomado Graduado en Economía y Gestión de la Construcción - Profesional Diploma / Degree in Construcción Economics and Management (Quantity Surveying). Se cursan en tres años de estudios académicos más un año de práctica. Por otro lado están: Diplomado / Graduado en Ingeniería Constructiva y Gestión de la Construcción - Professional Diploma/Degree in Building Engineering and Management. Y Licenciado en Gestión de la Construcción (Bachelor of Science in Construction Management).

\subsection{Italia}

La Enseñanza universitaria en Italia se estructura en dos ciclos o niveles. El primero, de tres años, permite acceder al título de Laurea, que faculta para la adquisición de competencias profesionales generales. El segundo ciclo, de dos años, conduce al título de Laurea Magistrale, y faculta para el ejercicio de actividades especializadas o de elevada cualificación.

Existe una amplia autonomía, pero se distinguen, básicamente, dos carreras universitarias relacionadas, con sus dos ciclos (en algún caso unificados), que intervienen en la construcción: Architettura, y , sobre todo, Ingegneria Edile (Ingeniería de Edificación), que, a veces, conforman una titulación doble. A ellas se puede añadir, con menor correspondencia, Ingegneria Civile. En muchas universidades, la orientación o especialización (diseño, paisaje, urbanismo, construcción, restauración, ...), se inicia desde el nivel de Laurea o grado. Los estudios de Arquitectura, en uno o dos ciclos, amplían la formación humanística, y están más vinculados al diseño, mientras que los de Ingeniería, más científicos, están más orientados a la tecnología y la producción.

\subsection{Luxemburgo}

Para poder llevar el título de Ingeniero en Luxemburgo se exige la homologación de un título superior correspondiente, adquirido en el extranjero en una carrera de al menos cuatro años y que, en el país donde se ha obtenido, dé derecho a cursar estudios de doctorado.

\subsection{Noruega}

Las formación universitaria se oferta en Noruega en dos modalidades básicas: por un lado están las carreras de ciclo largo, de, al menos, cuatro años y medio, que se estudian en los centros denominados Universitetet o Arkitelthogskolen; y, por otro, las carreras de ciclo corto, de orientación más práctica, y con una duración de dos o tres años, que, en el área técnica, se cursan en centros denominados Ingeniorhogskole. 
Son tres las formaciones universitarias, de tres años o más, que facultan en Noruega para un ejercicio profesional relacionado con la construcción: Arquitecto Civil (Sivilarkitekt), título de al menos cinco años, que se puede obtener, bien en una Escuela Superior de Arquitectura (Arkitekthogskolen), o bien en una Universidad Técnica (Norges Tekniske Hogskole); Ingeniero Civil - Facultad de la Construcción (Sivilingenior - Fakultet for Bygningsingeniorfag). Título de cuatro años y medio que se obtiene en una Universidad Técnica, y que incluye a todas las ramas de la Ingeniería, (mecánicos, químicos, eléctricos, etc.); y por último, Ingeniero de la Construcción (Ingenior, Avdeling Bygg og Anlegg). Literalmente "Ingeniero, Departamento de Construcción e Instalaciones", que se cursa en Escuelas de Ingeniería (Ingeniorhogskole).

\subsection{Países bajos}

El sistema neerlandés de Enseñanza Superior consta de cuatro tipos de establecimientos: las Universidades Técnicas, que forman a los ingenieros (especializados en Arquitectura o en Ingeniería Civil); las Escuelas Profesionales Superiores (Technische Hogescholen o Hogere Beroeps Onderwijs - HBO), que forman a los Ingenieros Técnicos (en Arquitectura o Ingeniería Civil); las Escuelas Técnicas Superiores (Hogere Technische Instituten - HTI), que permiten a los ingenieros técnicos acceder posteriormente al nivel de Ingeniero Civil; y las Academias de Bellas Artes (Academie van Bouwkunst), que permiten a los ingenieros técnicos acceder al título de Arquitecto.

Los titulados de las Escuelas Profesionales pueden acceder al nivel superior mientras están ejerciendo su profesión en el sector de la construcción, a condición de seguir los cursos de una Academia de Bellas Artes, para obtener el título de Arquitecto, o de una Escuela Técnica Superior, para obtener el título de Ingeniero. Vía de acceso esta tanto o mejor considerada que la vía directa de carácter universitario.

\subsection{Portugal}

En el sistema portugués los diferentes niveles de enseñanza están comunicados entre sí. De modo que la formación profesional y la formación politécnica, no universitaria, están comunicados con los estudios universitarios.

A nivel de estudios superiores en el sector de la construcción, se pueden distinguir dos carreras universitarias de tipo largo (5 años o más): Licenciado en Arquitectura y Licenciado en Ingeniería Civil; así como una carrera de tipo intermedio (3 años), de carácter politécnico: Ingeniero Técnico, en la especialidad de Ingeniería Civil, pero cuyos titulados pueden acceder a la Licenciatura universitaria mediante unos estudios complementarios de tres años. O pueden realizar un Diploma de Estudios Superiores Especializados (DESE), de una duración de dos años, que les permite obtener el título de Licenciado en Ingeniería Especializada (Dirección de Obras), que asimismo puede equipararse con el de Ingeniero Civil. 


\subsection{Reino unido}

El sistema educativo británico se distingue por la gran autonomía de los centros docentes para fijar sus programas y planes de estudios, y por la gran importancia que concede a los períodos de prácticas, previstos casi siempre como parte integrante de la formación. Esto permite una fácil adaptación a las necesidades del mercado de trabajo, pero provoca una gran dispersión de la oferta formativa, lo que dificulta la equiparación y homologación de los títulos académicos expedidos por las distintas universidades.

De este modo, los estudios relacionados son más diversos y especializados que en la mayoría de los países comunitarios. A ellos se puede acceder desde distintos títulos académicos de Bachelor of Science (BSc). Las carreras que representan, como mínimo, cuatro años de estudios superiores, incluidos los períodos de prácticas obligatorios, son: Arquitecto - Arquitect; Constructor - Builder; Experto en Construcción - Building Surveyor; Experto en Costes - Quantity Surveyor; Ingeniero de Estructuras - Civil and Structural Engineer; Ingeniero de Instalaciones - Building Services Engineer.

\subsection{Suecia}

En Suecia, los estudios superiores de ciclo largo en Ingeniería y Arquitectura, se realizan en la universidad y tienen una duración de cuatro años y medio o cinco años, incluyendo un periodos de prácticas de entre cuatro y seis meses. Las profesiones con formación universitaria relacionadas con la construcción son: Arquitecto (Arkitekt); Ingeniero Civil (Civilingenjör) que, aunque designa a todo tipo de ingenieros con formación de ciclo largo, suele aplicarse, por antonomasia, al Ingeniero de Caminos y Obras Hidráulicas (Väg- och vattenbyggnadsingenjör). Existe, no obstante, una amplia variedad de especializaciones (Ingeniería Estructural; Técnicas de Construcción de Edificios; Dirección de Proyectos de Edificación, o de Infraestructuras -Proyect Management-; Geotécnica; Ingeniería Hidráulica y Técnicas de Conservación del Agua; Construcción de Carreteras y Planificación del Tráfico; Planificación Territorial y Urbanismo; Ingeniería Ambiental). Por otra parte, la Escuela de Ingeniería (Ingenijörskola) de la Real Universidad Técnica de Estocolmo ofrece una carrera de tres años, que combina la ingeniería de la construcción con su gestión económica: Ingeniero en Técnica de Construcción y Economía (Ingenjör i Byggteknik och Ekonomi).

\section{La adaptación de los planes de estudio y la orden ECI}

Todas las universidades españolas, con mayor o menor premura, adaptaron sus planes de estudio a las nuevas determinaciones del Libro Blanco y del EEES. Del estudio anterior se infería directamente que el ámbito de estudio de los aquí denominados arquitectos técnicos era el de la ingeniería, sin que su ámbito de actuación y competencia fuera completamente ajeno a lo que había sido hasta ese momento. La titulación pasó a ser de grado, de nivel análogo al resto de titulaciones de grado, al margen de las pugnas profesionales, y dio acceso a estudios de segundo ciclo: másteres y doctorado de forma automática. 
Por tanto, lo que se aprobó en nuestras Escuelas fueron unos planes de estudios de Ingeniería, que preparan a profesionales perfectamente homologables en la esfera internacional. Pese a la desafortunada Sentencia que obligó al cambio de denominación del Título (aunque no en todos los Centros, no se olvide), los planes de estudios del Libro Blanco, cuyo contenido nunca ha sido cuestionado, siguen formando Ingenieros.

Es más, previamente a los recursos y a la Sentencia del Tribunal Supremo, que se verá más tarde, se había firmado un acuerdo entre el Consejo General de la Arquitectura Técnica CGATE, y el Consejo General de la Arquitectura CGA, con el visto bueno de los Ministerios de Educación y de Vivienda, aceptando todos, plenamente, la denominación de Ingeniero de Edificación para el título que se proponía en el Libro Blanco.

Con posterioridad se produce un hecho de gran trascendencia cual es la publicación de la Orden ECl-3855/2007, de 27 de diciembre, (que merecería más atención por sí sola), por la que se establecen los requisitos para la verificación de los títulos universitarios oficiales que habiliten para el ejercicio de la profesión de Arquitecto Técnico. Ello es, en lo que a su ejercicio regulado o habilitante, se refiere, claro está, que no al conjunto de su actividad profesional. Ha de tenerse en cuenta que, de acuerdo con datos de los Colegios, incluso durante el periodo en el que se desarrolló en España la burbuja inmobiliaria (1998-2007) que daría lugar a la crisis que finalmente se manifestó en toda su crudeza a partir de 2010 , el número de colegiados dedicados a la actividad profesional regulada no superó el $26 \%$ del total de egresados.

Como se ha dicho, las Escuelas de España invitaron a actuar, como un participante más, al CGATE en la elaboración del Libro Blanco. Sin embargo, sorprendentemente, en la discusión de dicha Orden, en la que sí intervino y activamente el CGATE, en ningún caso se invita a participar, ni se consulta a las universidades donde se impartían los títulos afectados. En otras titulaciones "habilitantes" fueron las universidades las que definieron las competencias reguladas de las titulaciones. No fue el caso para la Ingeniería de Edificación.

Y fue precisamente, un párrafo de esta Orden, el que establecía que el Ingeniero en Edificación era el único ingeniero habilitado para intervenir en edificación, el que provocó que los ingenieros en general, pero especialmente los Ingenieros Industriales y los Ingenieros Técnicos Industriales se opusieran frontalmente a dicha orden y la recurrieran. Aunque el resultado de su recurso tuvo otras consecuencias sobre la denominación.

\section{Y la sentencia del Supremo. Situación actual de la titulación y sus denominaciones en España}

Finalmente la conocida Sentencia del Tribunal Supremo (STS), haciendo una interpretación más allá de lo solicitado por las corporaciones recurrentes, determina que la denominación de la titulación induce a "confusión" al no corresponderse el título académico y profesional (el regulado o habilitante). Aunque no fue unánime, pues, como estableció el ex presidente del Tribunal Constitucional, D. Pascual Sala, en su voto particular a la Sentencia, son muy variadas las denominaciones académicas que no se corresponden con una titulación 
profesional. Sirva como ejemplo la denominación profesional de Abogado, cuya titulación académica era Licenciado en Derecho, o en la actualidad graduado/a en Derecho.

Ello condujo a las distintas universidades recurridas (que no fueron todas) y a algunas otras, en mayor o menor grado presionadas por sus rectores, algunos de ellos con la titulación de los recurrentes, a cambiar la denominación de las titulaciones de Ingeniería de Edificación. Y, paradójicamente, a un confuso conglomerado de nombres distintos para un mismo grado, que puede resumirse en:

- Graduado/a en Ingeniería de Edificación (mantenido en las universidades cuya denominación no fue recurrida)

- Graduado/a en Edificación

- Graduado/a en Arquitectura Técnica y Edificación

- Graduado/a en Arquitectura Técnica.

- Temporalmente, también hubo alguna denominación de Graduado/a en Ciencia y Tecnología de la Edificación.

Los planes de estudio de las distintas universidades, que siguieron el modelo del EEES a través del Libro Blanco, son, en todo caso, de ingeniería, nunca fueron recurridos en su contenido y nivel de formación que otorgan, por tanto, están plenamente vigentes, pese a la prohibición, para ciertas universidades del uso del término ingeniería. Por universidades y centros el catálogo de titulaciones vigente en la actualidad es el siguiente:

Tabla 1. Denominación de las titulaciones de grado por centro

\begin{tabular}{|c|c|c|}
\hline LOCALIDAD & CENTRO & $\begin{array}{l}\text { TITULACIONES QUE SE } \\
\text { IMPARTEN }\end{array}$ \\
\hline ALICANTE & $\begin{array}{l}\text { Escuela Politécnica Superior de } \\
\text { ALICANTE }\end{array}$ & $\begin{array}{lll}\text { GRADO } & \text { EN ARQUITECTURA } \\
\text { TECNICA } & & \end{array}$ \\
\hline BARCELONA & $\begin{array}{l}\text { Escuela Politécnica Superior de } \\
\text { Edificación (EPSB) de } \\
\text { BARCELONA. }\end{array}$ & $\begin{array}{l}\text { GRADO EN ARQUITECTURA } \\
\text { TÉCNICA Y EDIFICACIÓN }\end{array}$ \\
\hline $\begin{array}{l}\text { BARCELONA } \\
\text { PRIVADA }\end{array}$ & $\begin{array}{l}\text { UNIVERSIDAD RAMÓN } \\
\text { LLULL } \\
\text { Escuela Técnica Superior de } \\
\text { arquitectura }\end{array}$ & $\begin{array}{l}\text { GRADO EN ARQUITECTURA } \\
\text { TÉCNICA Y EDIFICACIÓN }\end{array}$ \\
\hline BURGOS & $\begin{array}{l}\text { Escuela Politécnica Superior de } \\
\text { BURGOS. }\end{array}$ & $\begin{array}{lll}\text { GRADO EN } & \text { ARQUITECTURA } \\
\text { TECNICA } & \\
\text { DOBLE GRADO EN INGENIERÍA } \\
\text { CIVIL Y Y ARQUITECTURA } \\
\text { TÉCNICA } & \\
\end{array}$ \\
\hline CACERES & $\begin{array}{l}\text { Escuela Politécnica Universidad } \\
\text { de EXTREMADURA }\end{array}$ & GRADO EN EDIFICACIÓN \\
\hline CARTAGENA & $\begin{array}{l}\text { Escuela de Arquitectura e } \\
\text { Ingeniería de Edificación } \\
\text { CARTAGENA }\end{array}$ & $\begin{array}{lll}\text { GRADO EN } & \text { INGENIERÍA } & \text { DE } \\
\text { EDIFICACIÓN } & & \\
\end{array}$ \\
\hline
\end{tabular}




\begin{tabular}{|c|c|c|}
\hline LOCALIDAD & CENTRO & $\begin{array}{l}\text { TITULACIONES QUE SE } \\
\text { IMPARTEN }\end{array}$ \\
\hline CASTELLÓN & $\begin{array}{l}\text { Escuela Superior de Tecnología } \\
\text { y Ciencias Experimentales. } \\
\text { CASTELLÓN PLANA }\end{array}$ & $\begin{array}{lll}\text { GRADO } & \text { EN } & \text { ARQUITECTURA } \\
\text { TECNICA } & & \end{array}$ \\
\hline CUENCA & $\begin{array}{lll}\text { Escuela } & \text { Politécnica } & \text { de } \\
\text { CUENCA } & & \\
\end{array}$ & $\begin{array}{lcc}\text { GRADO EN } & \text { INGENIERÍA } & \text { DE } \\
\text { EDIFICACIÓN } & & \end{array}$ \\
\hline GIRONA & $\begin{array}{l}\text { Escuela Politécnica Superior } \\
\text { GERONA }\end{array}$ & $\begin{array}{l}\text { GRADO EN ARQUITECTURA } \\
\text { TÉCNICA Y EDIFICACIÓN }\end{array}$ \\
\hline GRANADA & $\begin{array}{l}\text { Escuela Técnica Superior de } \\
\text { Ingeniería de Edificación } \\
\text { GRANADA. }\end{array}$ & $\begin{array}{l}\text { GRADO EN EDIFICACIÓN } \\
\text { DOBLE GRADO } \\
\text { EDIFICACIÓN Y ADE }\end{array}$ \\
\hline GUADALAJARA & Escuela de Arquitectura & $\begin{array}{l}\text { GRADO EN ARQUITECTURA } \\
\text { TÉCNICA Y EDIFICACIÓN }\end{array}$ \\
\hline $\begin{array}{l}\text { ISLAS } \\
\text { BALEARES }\end{array}$ & $\begin{array}{l}\text { Escuela Politécnica Superior. } \\
\text { ISLAS BALEARES }\end{array}$ & GRADO EN EDIFICACIÓN \\
\hline LA CORUÑA & $\begin{array}{l}\text { Escuela Universitaria de } \\
\text { Arquitectura Técnica } \\
\text { LA CORUÑA }\end{array}$ & $\begin{array}{lll}\text { GRADO } & \text { EN ARQUITECTURA } \\
\text { TÉCNICA } & & \end{array}$ \\
\hline MADRID & $\begin{array}{l}\text { Escuela Técnica Superior de } \\
\text { Edificación } \\
\text { MADRID }\end{array}$ & $\begin{array}{l}\text { GRADO EN EDIFICACIÓN } \\
\text { GRADO EN EDIFICACIÓN + ADE }\end{array}$ \\
\hline $\begin{array}{l}\text { MADRID } \\
\text { PRIVADA }\end{array}$ & $\begin{array}{l}\text { UNIVERSIDAD ALFONSO X } \\
\text { EL SABIO } \\
\text { Escuela Politécnica Superior. }\end{array}$ & GRADO EN EDIFICACIÓN \\
\hline $\begin{array}{l}\text { MADRID } \\
\text { PRIVADA }\end{array}$ & $\begin{array}{l}\text { UNIVERSIDAD CAMILO JOSÉ } \\
\text { CELA } \\
\text { Escuela Supr. Arquitectura y } \\
\text { Tecnología (ESAT). }\end{array}$ & NO IMPARTE \\
\hline $\begin{array}{l}\text { MADRID } \\
\text { Villaviciosa de } \\
\text { Odón } \\
\text { PRIVADA }\end{array}$ & $\begin{array}{l}\text { UNIVERSIDAD EUROPEA DE } \\
\text { MADRID. } \\
\text { Escuela Superior de Arte y } \\
\text { Arquitectura. }\end{array}$ & NO IMPARTE \\
\hline $\begin{array}{l}\text { MURCIA } \\
\text { PRIVADA }\end{array}$ & $\begin{array}{l}\text { UNIVERSIDAD } r \text { CATÓLICA } \\
\text { SAN ANTONIO } \quad \text { Escuela } \\
\text { Universitaria Politécnica. }\end{array}$ & $\begin{array}{l}\text { GRADO EN INGENIERÍA DE } \\
\text { EDIFICACIÓN }\end{array}$ \\
\hline SEVILLA & $\begin{array}{l}\text { Escuela Técnica Superior de } \\
\text { Ingeniería de Edificación } \\
\text { SEVILLA }\end{array}$ & GRADO EN EDIFICACIÓN \\
\hline LA LAGUNA & $\begin{array}{l}\text { Escuela Politécnica Superior } \\
\text { Ingeniería de LA LAGUNA }\end{array}$ & $\begin{array}{l}\text { GRADO EN } \\
\text { TÉCNICA }\end{array}$ \\
\hline LLEIDA & $\begin{array}{l}\text { Escuela Politécnica Superior } \\
\text { LLEIDA }\end{array}$ & $\begin{array}{l}\text { GRADO EN ARQUITECTURA } \\
\text { TÉCNICA y EDIFICACIÓN }\end{array}$ \\
\hline SAN SEBASTIÁN & $\begin{array}{l}\text { Escuela Universitaria } \\
\text { Politécnica de Donostia }\end{array}$ & $\begin{array}{l}\text { GRADO EN } \\
\text { TÉCNICA }\end{array}$ \\
\hline
\end{tabular}


Juan M. Santiago Zaragoza, Fabián García Carrillo, Joaquín Passolas Colmenero, Emilio Gómez Cobos

\begin{tabular}{|c|c|c|}
\hline LOCALIDAD & CENTRO & $\begin{array}{l}\text { TITULACIONES QUE SE } \\
\text { IMPARTEN }\end{array}$ \\
\hline VALENCIA & $\begin{array}{l}\text { Escuela Técnica Superior de } \\
\text { Ingeniería de Edificación } \\
\text { VALENCIA }\end{array}$ & $\begin{array}{lll}\text { GRADO EN ARQUITECTURA } \\
\text { TÉCNICA }\end{array}$ \\
\hline $\begin{array}{l}\text { VALLADOLID } \\
\text { PRIVADA }\end{array}$ & $\begin{array}{l}\text { UNIVERSIDAD } \quad \text { EUROPEA } \\
\text { MIGUEL DE CERVANTES } \\
\text { Escuela Politécnica Superior }\end{array}$ & $\begin{array}{l}\text { GRADO EN ARQUITECTURA } \\
\text { TÉCNICA }\end{array}$ \\
\hline ZAMORA & $\begin{array}{l}\text { Escuela Politécnica Superior de } \\
\text { ZAMORA. }\end{array}$ & $\begin{array}{l}\text { GRADO EN ARQUITECTURA } \\
\text { TÉCNICA }\end{array}$ \\
\hline ZARAGOZA & $\begin{array}{l}\text { Escuela } \\
\text { Politécnica } \\
\text { ZARAGOZA }\end{array}$ & $\begin{array}{lll}\text { GRADO } & \text { EN ARQUITECTURA } \\
\text { TÉCNICA } & & \\
\end{array}$ \\
\hline
\end{tabular}

\section{La situación actual y el futuro de la titulación.}

\subsection{El incremento de competencias en los grados}

Al margen de las diferentes denominaciones de la titulación académica, es una realidad palpable que todas ellas habilitan para el ejercicio profesional regulado de la Arquitectura Técnica. $Y$ así debe seguir siendo mientras dichas reservas sigan existiendo en España. La situación en los diferentes países de la Unión Europea nos muestra una realidad completamente diferente, para la que habrá que estar preparado.

Sobre la base de los 240 créditos ECTS del Grado universitario, de los cuales la Orden ECI, fijó los créditos de carácter Básico en 60, los de Proyecto de Fin de Grado en 12, y limitó los de Formación Específica a 108, dejando pues, 60 créditos optativos para ser determinados, en sus contenidos y competencias, por las distintas Universidades. Esto acabaría permitiendo la intensificación de ciertas áreas o la especialización en cada Plan de Estudios concreto, es decir, la adquisición de nuevas competencias, complementarias a la formación contenida en los 180 créditos comunes de la Orden $\mathrm{ECl}$, que, en definitiva, son los que deben concentrar y otorgar las competencias establecidas para la parte regulada, o habilitante, para el ejercicio de la profesión de Arquitecto Técnico. Competencias que el Grado, con sus 240 créditos, lógicamente supera. Por tanto, con todos los respetos, no cabe una identificación como la que se alude en la sentencia.

\subsection{Y la formación de posgrado}

Prácticamente todas las universidades españolas han implantado un extenso catálogo de títulos de máster a los que (obviados los másteres habilitantes) tienen acceso los graduados de las titulaciones aquí mencionadas. Más aún, muchas de nuestras Escuelas han implantado programas de máster diseñados para la especialización de nuestros graduados. Dichos títulos oficiales, con reconocimiento inmediato a nivel europeo y, por extensión a nivel mundial, ofrecen nuevas competencias a nuestros egresados que deben trasladarse a su campo de actividad profesional. 
Los Másteres, antes inaccesibles, son una feliz realidad, y ofrecen a los nuevos titulados extraordinarias posibilidades para continuar y ampliar la formación regulada, por la vía de la especialización, otorgando nuevas competencias que, indudablemente, les sitúan en un nivel de cualificación avanzado, también en el ámbito de la Ingeniería. Los colegios profesionales, tradicionalmente, han apostado por los cursos de formación, como forma de mejorar en el ejercicio profesional, extendiéndolo así a nuevas facetas de actividad. No sería razonable que estos mismos colegios, ahora, estén dispuestos a cuestionar la formación de posgrado cuando, además, tiene carácter oficial y nivel universitario. Incluso las Escuelas de Arquitectura (cuyo título oficial es un Máster habilitante con atribuciones por encima de toda lógica), ofrecen Másteres de especialización que, evidentemente, aportan nuevas competencias y abren diversos campos al ejercicio de esa profesión.

El presente y el futuro, -desde la UE ya se nos viene avisando-, más que en las atribuciones reguladas, está en el ejercicio de las competencias reales, y en la asunción de las responsabilidades efectivas por cada cual. Y para ello hay que prepararse. La Universidad, nuestras Escuelas, van a seguir apostando por la ampliación de competencias a través de los Másteres, y los Colegios deben entenderlos como una vía para una ampliación cualitativa de su campo de actividad, además de como una oportunidad de colaboración más estrecha con la parte académica.

Las Escuelas generan, articulan o participan en una oferta variada y nada desdeñable de Másteres (Tabla 2) que ya están a disposición de los graduados y que, fundamentalmente, se sustentan en nuestras propias capacidades para proporcionar una oferta de formación atractiva. En estos Másteres suelen participar profesionales de reconocido prestigio ajenos a la Universidad, por tanto, pueden ser un extraordinario nexo de colaboración entre Academia y Profesión. De hecho, esa colaboración ya se está produciendo.

Tabla 2. Denominación de las titulaciones de máster por centro

\begin{tabular}{|l|l|l|}
\hline \multicolumn{1}{|c|}{ LOCALIDAD } & \multicolumn{1}{|c|}{ CENTRO } & \multicolumn{1}{|c|}{ MÁSTERES QUE SE IMPARTEN } \\
\hline \hline BARCELONA & $\begin{array}{l}\text { Escuela Politécnica Superior } \\
\text { de Edificación (EPSB) de } \\
\text { BARCELONA. }\end{array}$ & $\begin{array}{l}\text { Máster universitario en Construcción } \\
\text { Avanzada en la Edificación. } \\
\text { Máster universitario en Diagnosis y } \\
\text { Técnicas de Intervención en la } \\
\text { Edificación } \\
\text { Máster universitario en Gestión de la } \\
\text { Edificación }\end{array}$ \\
\hline
\end{tabular}




\begin{tabular}{|c|c|c|}
\hline LOCALIDAD & CENTRO & MÁSTERES QUE SE IMPARTEN \\
\hline $\begin{array}{l}\text { BARCELONA } \\
\text { PRIVADA }\end{array}$ & $\begin{array}{l}\text { UNIVERSIDAD RAMÓN } \\
\text { LLULL } \\
\text { Escuela Técnica Superior } \\
\text { de arquitectura }\end{array}$ & 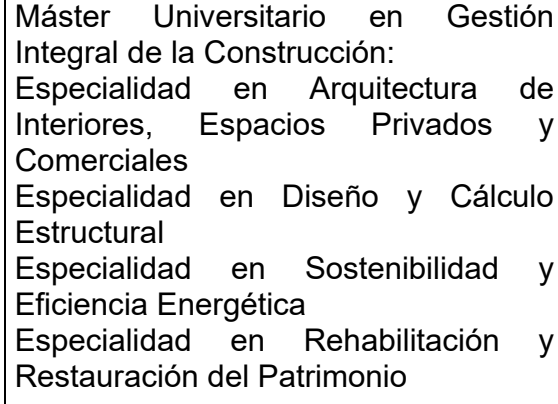 \\
\hline BURGOS & $\begin{array}{l}\text { Escuela Politécnica Superior } \\
\text { de BURGOS. }\end{array}$ & \begin{tabular}{|lrl} 
Máster Universitario en Inspección, \\
Rehabilitación y Eficiencia Energética \\
en la Edificación & \\
Máster Universitario en & Integridad y \\
Durabilidad de & Materiales, \\
Componentes y & Estructuras \\
(Interuniversitario; & \\
streaming/presencial) &
\end{tabular} \\
\hline CACERES & $\begin{array}{l}\text { Escuela Politécnica } \\
\text { Universidad de } \\
\text { EXTREMADURA }\end{array}$ & $\begin{array}{l}\text { Máster Universitario de Metodología } \\
\text { para Modelización de la Información } \\
\text { Construcción (Bim) }\end{array}$ \\
\hline CARTAGENA & $\begin{array}{l}\text { Escuela Arquitectura e } \\
\text { Ingeniería de Edificación } \\
\text { CARTAGENA }\end{array}$ & $\begin{array}{l}\text { Máster en Ciencia y Tecnología de la } \\
\text { Edificación en Arquitectura } \\
\text { Máster en Patrimonio Arquitectónico }\end{array}$ \\
\hline CASTELLÓN & $\begin{array}{l}\text { Escuela Superior de } \\
\text { Tecnología y Ciencias } \\
\text { Experimentales. } \\
\text { CASTELLÓN PLANA }\end{array}$ & $\begin{array}{l}\text { Máster en Eficiencia Energética y } \\
\text { Sostenibilidad }\end{array}$ \\
\hline GRANADA & $\begin{array}{l}\text { Escuela Técnica Superior } \\
\text { de Ingeniería de Edificación } \\
\text { GRANADA. }\end{array}$ & $\begin{array}{l}\text { Máster en Ciencia y Tecnología del } \\
\text { Patrimonio Arquitectónico (CITPA) } \\
\text { Máster en Rehabilitación } \\
\text { Arquitectónica } \\
\text { Máster en Estructuras } \\
\text { Máster en Prevención de Riesgos } \\
\text { Laborales } \\
\text { Doble Máster en Rehabilitación } \\
\text { Arquitectónica + Estructuras } \\
\text { Doble Máster en Rehabilitación } \\
\text { Arquitectónica y CITPA }\end{array}$ \\
\hline GUADALAJARA & Escuela de Arquitectura & $\begin{array}{l}\text { Máster Universitario en Gestión } \\
\text { Integral de Inmuebles y Servicios en el } \\
\text { Patrimonio Arquitectónico }\end{array}$ \\
\hline
\end{tabular}




\begin{tabular}{|c|c|c|}
\hline LOCALIDAD & CENTRO & MÁSTERES QUE SE IMPARTEN \\
\hline LA CORUÑA & $\begin{array}{l}\text { Escuela Universitaria de } \\
\text { Arquitectura Técnica } \\
\text { LA CORUÑA }\end{array}$ & $\begin{array}{l}\text { Máster Universitario en } \\
\text { integrada de proyectos } \\
\text { Máster Universitario en Edificación } \\
\text { Sostenible }\end{array}$ \\
\hline MADRID & $\begin{array}{l}\text { Escuela Técnica } \text { Superior } \\
\text { de Edificación } \\
\text { MADRID }\end{array}$ & $\begin{array}{l}\text { Máster en Innovación Tecnológica en } \\
\text { Edificación (MITE) } \\
\text { Máster en Gestión en Edificación } \\
\text { (MAGE) } \\
\text { Máster en Ejecución de Obras de } \\
\text { Rehabilitación y Restauración } \\
\text { (MEJORR) } \\
\text { Doble Máster en Innovación } \\
\text { Tecnológica en Edificación y Máster } \\
\text { en Ejecución de Obras de } \\
\text { Rehabilitación y Restauración }\end{array}$ \\
\hline $\begin{array}{l}\text { MURCIA } \\
\text { PRIVADA }\end{array}$ & $\begin{array}{l}\text { UNIVERSIDAD CATÓLICA } \\
\text { SAN ANTONIO Escuela } \\
\text { Universitaria Politécnica. }\end{array}$ & $\begin{array}{l}\text { Máster Universitario en Patología e } \\
\text { Intervención en Edificación }\end{array}$ \\
\hline SEVILLA & $\begin{array}{l}\text { Escuela Técnica Superior } \\
\text { de Ingeniería de Edificación } \\
\text { SEVILLA }\end{array}$ & $\begin{array}{l}\text { Máster Universitario en Seguridad } \\
\text { Integral en Edificación (Itinerarios) } \\
\text { Máster Universitario en Gestión } \\
\text { Integral en Edificación (Itinerarios) }\end{array}$ \\
\hline LA LAGUNA & $\begin{array}{l}\text { Escuela Politécnica Superior } \\
\text { Ingeniería de LA LAGUNA }\end{array}$ & $\begin{array}{l}\text { Máster Universitario en Gestión e } \\
\text { Innovación Tecnológica en la } \\
\text { Construcción }\end{array}$ \\
\hline SAN SEBASTIÁN & $\begin{array}{|ll|}\text { Escuela } & \text { Universitaria } \\
\text { Politécnica de Donostia }\end{array}$ & $\begin{array}{l}\text { Máster en Ingeniería de Materiales } \\
\text { Renovables }\end{array}$ \\
\hline VALENCIA & $\begin{array}{l}\text { Escuela Técnica Superior } \\
\text { de Ingeniería de Edificación } \\
\text { VALENCIA }\end{array}$ & Máster en Edificación \\
\hline ZAMORA & $\begin{array}{l}\text { Escuela Politécnica Superior } \\
\text { de ZAMORA. }\end{array}$ & $\begin{array}{l}\text { Máster en Dirección y Gestión de } \\
\text { Proyectos } \\
\text { Máster en gestión integrada de } \\
\text { edificación y obra civil } \\
\text { Máster en Energías Renovables y } \\
\text { Eficiencia Energética }\end{array}$ \\
\hline
\end{tabular}

Por otra parte, y viendo una oportunidad en cada problema, el terrible y extraordinario suceso de la pandemia del coronavirus Covid-19 ha provocado, también, la obligada y súbita inmersión de la universidad española en la docencia on line. Los estudiantes reciben clases y tutorías a través de video conferencia, o clases grabadas previamente, que el profesor imparte y gestiona con la ayuda de una serie de aplicaciones y plataformas en la web. Este sistema, asimismo, puede ser idóneo para acercar nuestra oferta formativa a los profesionales en ejercicio con dificultades de acceso directo a la Universidad, pero que 
desean adaptar su título o realizar un posgrado. Desde la Conferencia de Directores de Arquitectura Técnica e Ingeniería de Edificación, CODATIE, y con ese específico fin, se hace una apuesta decidida por extender la formación on line, y en lo posible, la oferta académica actual de nuestras Escuelas, ampliándola incluso con propuestas de Másteres Interuniversitarios, en áreas y ámbitos de especialización donde no haya oferta suficiente.

\subsection{Del Doctorado y la carrera académica.}

La oferta universitaria, aún escasa, de Programas de Doctorado vinculados a nuestro específico campo de acción, posibilita el acceso de titulados Máster al máximo nivel de formación académica, y por tanto de especialización, que existe en la Universidad. El Doctorado es hoy la vía imprescindible para iniciar una carrera académica universitaria, pero no sólo es eso. No se nos puede escapar que también abre las puertas a nuestros profesionales para que ocupen puestos de la máxima responsabilidad en instituciones y empresas. De hecho, aunque aún no son muy numerosos, algunos de ellos empiezan a ostentar cargos directivos relevantes, en unidades de I+D+i de centros de investigación, de grandes empresas y administraciones. Colegios profesionales y universidades debemos sentirnos orgullosos de ellos pues son los mejores heraldos de nuestra formación y nuestra profesión, los más dignos representantes de nuestro colectivo.

Este indiscutible avance desde el lado académico, con clara y directa repercusión en el mundo profesional tampoco puede estar lastrado por una denominación previa de Grado que nos retrotraería a un tiempo en que esto era imposible. Un nombre distintivo y homologable, que reconoce en él el acceso a un nivel máximo de formación y responsabilidad, facilitará, en el futuro, incrementar aún más nuestra presencia y nuestro nivel de participación en órganos de gestión y decisión, en cualquier parte del mundo, en la esfera pública y privada. $Y$ poder, por fin, actuar con criterio propio, representativo de un colectivo profesional independiente, que análogamente a nuestros colegas del resto del mundo, ejerce e impone su "superior", por mejor, criterio.

Respecto a la carrera académica, no cabe dudar de la importancia y trascendencia que tiene para el futuro de nuestras Escuelas y Colegios, el que nuestros egresados accedan al Doctorado. Hasta ahora, si nuestros propios titulados están presentes entre el profesorado estable de las Escuelas, es porque, con un esfuerzo encomiable, han realizado otros estudios y leído tesis doctorales, iniciando la larga carrera de obstáculos que supone la acreditación a los distintos cuerpos universitarios. En algunas universidades se ha llegado a los puestos de alta responsabilidad en las Escuelas y la propia Universidad.

Pero no hay que llevarse a engaño, este no es, ni mucho menos, el panorama general. Hoy día, hay Centros que imparten los grados aquí mencionados en los que sólo un profesor, de todo el claustro, tiene la titulación de Arquitecto Técnico o Ingeniero de Edificación. Es fácil comprender que la visión de nuestra profesión, el diseño de los planes de estudios, la defensa de nuestros intereses, y hasta el enfoque de asignaturas "clave" en la formación de los titulados, dependen de la formación previa del profesorado. Consecuentemente, los Colegios, que se nutren de las Escuelas, deben adoptar una posición común ¡de máxima alerta!, y con la participación directa del CGATE y la misma CODATIE, elaborar y poner en 
práctica un plan estratégico, para fomentar que nuestros mejores estudiantes y egresados, puedan hacer carrera universitaria. El futuro de esta profesión estará en sus manos. Pero hoy está en las nuestras el que eso sea posible: no nos atrincheremos esperando a que el pasado venga a rescatarnos.

\section{Conclusiones}

\subsection{Un presente indudablemente mejor y un futuro sin vuelta atrás}

A lo largo de toda nuestra historia, como colectivo, tanto la formación de los titulados como la actividad profesional desarrollada, es decir, las competencias adquiridas y acreditadas, han superado, con creces, al angosto límite de las atribuciones reguladas en España. La conquista de nuevos campos de actividad en el cada vez más complejo mundo de la Edificación nunca ha supuesto un problema para un profesional dedicado precisamente a resolverlos. Se ha de reconocer con orgullo que, sin nuestra participación, este sector no funcionaría. Sin embargo, cuando se ha intentado el reconocimiento oficial de la actividad realizada, el intento ha devenido en un proceso tortuoso que, casi siempre ha encallado por la voluntad y el poder corporativo de otros que han sentido amenazados unos privilegios, legales sin duda, fundamentados en la asunción de unas supuestas máximas responsabilidades que, en algún caso, no se corresponden ni con la formación recibida ni con el ejercicio desarrollado.

El colectivo académico, con la participación del CGATE, ha contemporizado o ha consentido este statu quo más allá de lo admisible. Se ha de provechar el cambio y el reto que ha supuesto contrastar nuestras capacidades o habilidades, a escala europea y mundial, para darle un impulso a esta extraordinaria e imprescindible profesión, con unos fundamentos sólidos, y que está recuperando la buena coyuntura en lo laboral y que, sin lugar a duda alguna, sí que es el mejor momento que nunca ha vivido en lo académico. Aprovechemos nuestras sinergias, ¡Ni un paso atrás!

La CODATIE ha comunicado formalmente a la Conferencia de Rectores de las Universidades Europeas y al Ministerio de Universidades, el sentir manifestado por las Escuelas y Universidades españolas que imparten la titulación, comprometidas en la construcción del EEES, en el sentido de apostar decididamente por unificar la denominación del título universitario como Grado en Ingeniería de Edificación, que asimismo da lugar a la profesión del igual nombre, ambos homologables tanto en el EEES como a nivel mundial.

El Título permitirá el acceso a distintos niveles de formación universitaria de posgrado, en dicho campo de especialidad propio. Todo ello, de acuerdo con los criterios y directrices del Libro Blanco del Título de Grado en Ingeniería de Edificación aprobado por la ANECA, incluyéndose entre las competencias -no exclusivas- otorgadas por el Grado, las que habilitan para el ejercicio regulado en España de la anterior profesión de Arquitecto Técnico.

\section{¡Larga vida a la Ingeniería de Edificación!}




\section{Referencias}

AA..VV. (2005). Libro Blanco del Título de Grado en Ingeniería de Edificación. Agencia Nacional de Evaluación de la Calidad y Acreditación. Desk impresores S.L. Madrid

MARIA PALLISERA DÍAZ, M., FULLANA NOELL, J., PLANAS LLADÓ, A. Y DEL VALLE GÓMEZ, A., (2010), "La adaptación al espacio europeo de educación superior en España. Los cambios/retos que implica la enseñanza basada en competencias y orientaciones para responder a ellos". Revista Iberoamericana de Educación / Revista Ibero-americana de Educação. n. ${ }^{\circ}$ 52/4 - 25/04/10 